DOI: $10.7242 / 1998-2097 / 2018.1 .1$

УДК 519.6;536.2

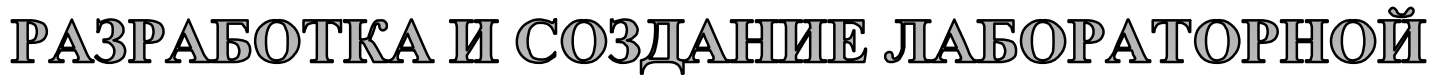

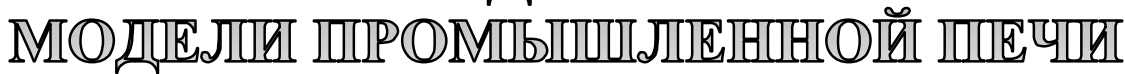

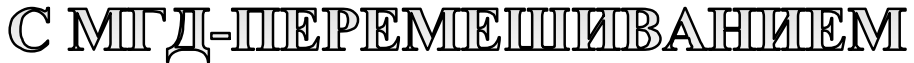

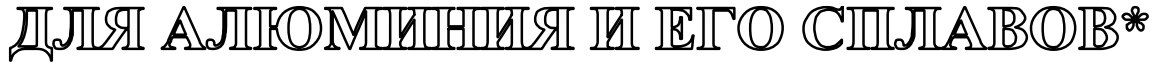

С.Ю. Хрипченко, Институт механики сплошных сред УрО РАН

С.А. Денисов, Институт механики сплошных сред УрО РАН

В.М. Долгих, Институт механики сплошных сред УрО РАН

С.В. Лекомцев, Институт механики сплошных сред УрО РАН

А.М. Павлинов, Институт механики сплошных сред УрО РАН

П.А. Оборин, Институт механики сплошных сред УрО РАН

В работе на фризических моделях ванны алюминиевой печи исследуется процесс перемешивания жидкого металла, бегущим магнитным полем, которое создается системой параллельных линейных проводников, расположенных под днищем ванны. На галлиевой модели с помощью допплеровского анемометра получены профили скорости течения и зависимость скорости перемешивания от силы и частоты тока в индукторе. В эксперименте обнаружено резкое уменьшение вертикального градиента температуры в ванне при достижении порогового значения скорости перемешивания жидкого металла. При помощи математического моделирования рассчитывается картина течения, эволюция поля температур и распространение примеси в ванне физической модели под действием МГД-перемешивания. Результаты сравниваются с результатами фризических экспериментов.

Ключевые слова: алюминиевая печь, МГД-перемешивание, бегущее поле, линейный индуктор, пассивная примесь, время перемешивания, градиент температуры.

1. Введение. Процесс гомогенизации среды при приготовлении сплавов является одной из наиболее исследуемых проблем для различных приложений [3-8]. Высокая температура расплавленных металлов приводит к тому, что бесконтактные методы, использующие МГД-перемешивание, становятся предпочтительнее прочих. Применяемые в настоящее время в алюминиевых печах МГД-перемешиватели в большинстве своем являются индукторами бегущего поля. Они много меньше размеров ванны, их полюса значительно удалены от жидкого металла вследствие большой толщины стенок и теплоизоляции ванны. Магнитное поле по этим причинам сильно ослаблено и воздействует на небольшой объем металла в ванне. Это приводит к тому, что они потребляют сотни кВА для создания необходимой интенсивности перемешивания металла. Целью наших исследований являлся поиск конструкции устройства перемешивания металла в алюминиевой печи, по возмож-

* Работа выполнена при поддержке гранта РФФИ № 14-08-96002. 
ности, лишенного этих недостатков. Нами предлагается конструкция, в которой индуктор бегущего поля представляет собой систему параллельных линейных проводников (труб) с текущим по ним переменным током, значительно приближенных к жидкому металлу. Трубы подключены к трехфазному источнику питания так, чтобы магнитное поле, создаваемое токами в трубах, было бегущим. Трубы проходят через теплоизоляционный слой под подиной ванны и приближены к жидкому металлу (рис. 1). В этом случае магнитное поле охватывает большую область ванны. Охлаждение труб осуществляется за счет термогравитационной конвекции [4].

2. Физические модели. Для исследования предлагаемого устройства мы разработали и создали лабораторные модели ванн с МГД-перемешиванием (рис. 2-4), на которых провели ряд экспериментальных и теоретических исследований процесса МГД-перемешивания жидкого металла.

Для иллюстрации процесса перемешивания был проведен демонстрационный эксперимент на электролитной модели, в которой в придонном слое через электролит пропускался постоянный электрический ток, а на дне кюветы располагались постоянные магниты, создающие вертикально направленное магнитное поле. При взаимодействии магнитного поля и электрического тока в придонном слое возникала электромагнитная сила, направленная вдоль дна и создающая перемешивающее течение в кювете (см. рис. 4).

В «галлиевой» модели (см. рис. 3) бегущее магнитное поле создает в придонном слое жидкого металла объемные силы, вызывающие в ванне перемешивающее течение. Эти силы, как показывают численные расчеты, зависят как от величины тока в токопроводах и его частоты, так и от расположения токопроводов относительно друг друга и от расстояния до жидкого металла в ванне (рис. 5, 6).

3. Течение жидкого металла в ванне под действием электромагнитных сил, создаваемых индуктором (для скоростей много меньших скорости бегущего поля), описывается безразмерными уравнениями Навье-Стокса и уравнениями электродина-

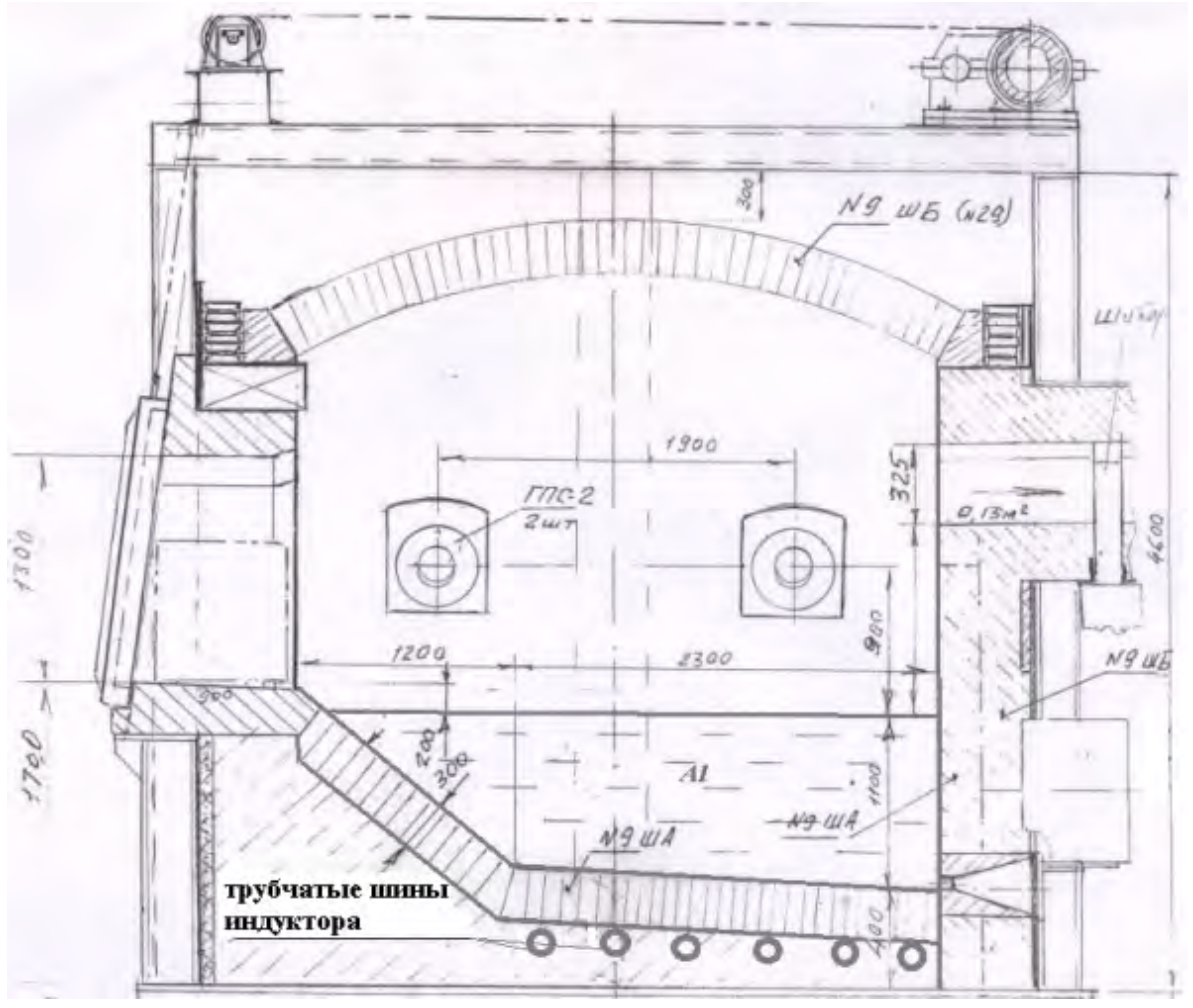

Рис. 1. Промышленная отражательная печь, работающая на газе, который, сгорая над поверхностью металла, плавит его. Под днищем печи трубчатые иины, создающие бегущее магнитное поле 
мики без учета движения среды $[2,7]$ :

$$
\left\{\begin{array}{l}
\frac{\partial \vec{V}}{\partial t}+(\vec{V} \nabla) \vec{V}=-\nabla p+\Delta \vec{V}+S \cdot \vec{j} \times \vec{B}, \\
\operatorname{div} \vec{V}=0, \quad \frac{\partial \vec{B}}{\partial t}=\frac{1}{\delta} \Delta \vec{B},
\end{array}\right.
$$

где $\quad S=f \cdot h^{3} / \rho v^{2}=j_{0} B_{0} h^{3} / \rho v^{2}=$ $=\sigma \omega \mu_{0}^{2} h^{4} I_{0}^{2} / 4 \rho v^{2} \pi^{2} R_{0}^{2}, \quad \delta=h^{2} \sigma \mu_{0} \omega, \quad \sigma-$

электрическая проводимость жидкого металла, $\omega$ - циклическая частота тока в ин-

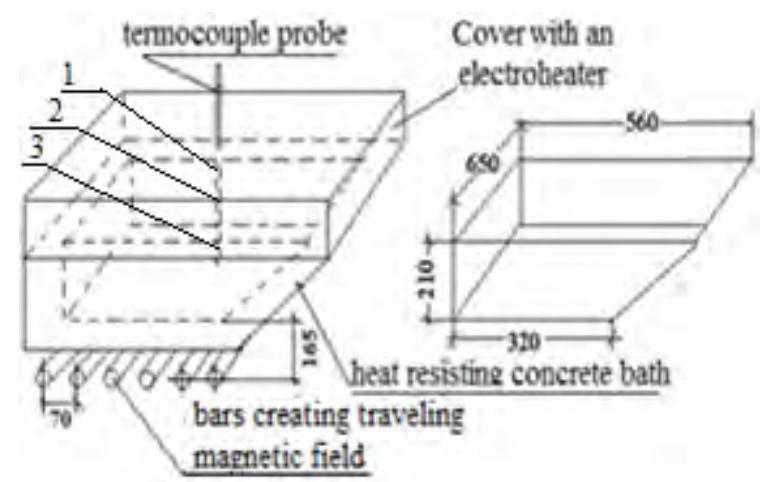

Рис. 2. Модель ванны алюминиевой печи из жаропрочного бетона (с алюминием в качестве рабочего тела). Термопарные спаи располагались в точке 1 на глубине 32 мм от поверхности слоя металла, в точке 2 на глубине 57 мм, в точке 3 - на глубине 83 мм

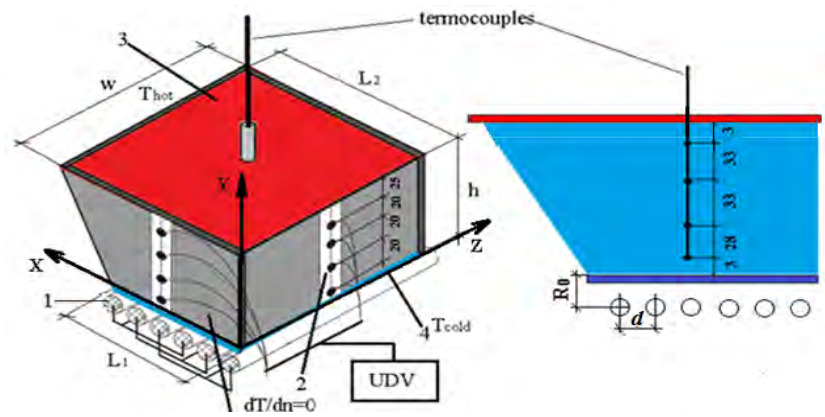

$a$

$\sigma$

Рис. 3. Модель ванны алюминиевой печи (с галлием в качестве рабочего тела) (а) и схема расположения спаев термопар в ванне (б). Крышка и дно ванны поддерживались при постоянной температуре соответственно $T_{\text {hot }}=32^{\circ} \mathrm{C}, T_{\text {cold }}=22^{\circ} \mathrm{C}$. Стенки ванны из нержавеющей стали толщиной 1 мм. Боковые стенки теплоизолированы. Ванна заполнена галлиевым сплавом. $H=100$ мм, $w=340$ мм, $L_{1}=180$ мм, $L_{2}=240$ мм. Под днищем расположены линейные токопроводы, создающие бегущее магнитное поле $R_{0}=30$ мм, $d=25$ мм. На боковых стенках расположень датчики скорости ультразвукового допплеровского анемометра. 1 -иины индуктора; 2 - датчики допплеровского анемометра; 3 - крышка с нагревателем; 4 -дно с холодильником дукторе, $\mu_{0}-$ магнитная проницаемость вакуума, $h$ - толщина слоя металла, $I_{0}-$ ток в шине индуктора, $\rho-$ плотность металла, $v$ - кинематическая вязкость, $R_{0}-$ расстояние шин индуктора до слоя металла в ванне. За масштаб размера и скорости выбраны соответственно $h$ и $v / h$.

При расчете течения жидкого металла в ванне граничные условия соответствовали условиям прилипания и непротекания на стенках области. Система уравнений (1) решалась конечно-разностным способом в естественных переменных методом расщепления [1], который состоит в последовательном учете нелинейных членов, вязкости, давления и электромагнитной силы. Для описания турбулентности использовалась $k-\varepsilon$ модель. Сетка имела $500 \times 250$ узлов.

Расчеты течения в галлиевой модели при токе индуктора 200 А показали, что течение представляет собой один большой
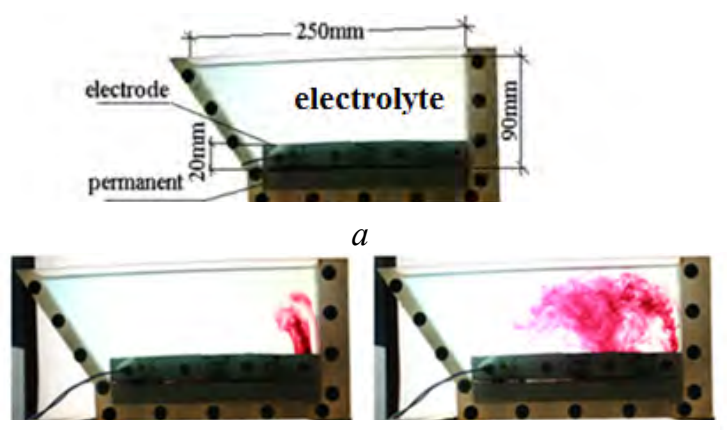

$\sigma$

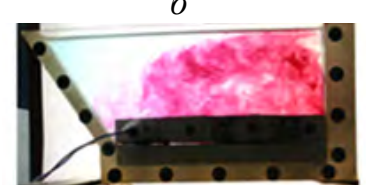

2

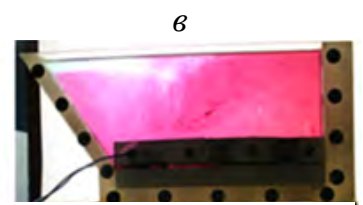

$\partial$
Рис. 4. Модель с раствором $\mathrm{CuSO}_{4}$ для визуализации процесса перемешивания: $a$ - высота слоя электролита 90 мм, толщчина слоя 20 мм. Внизу в придонной области находится слой подкрашенного электролита; б, в, г, д-картины перемешивания пассивной примеси при включении электромагнитных сил (картинки сняты с интервалом в 15 с). Средняя вертикальная составляющая магнитного поля в области электродов составляет 86 мТл.

Через слой электролита

в придонной области протекает ток в 1,5 A.

Средняя горизонтальная составляющая

скорости перемешивания в верхней части кюветь $6,9 \pm 0,6 \mathrm{MM} / \mathrm{c}$ 


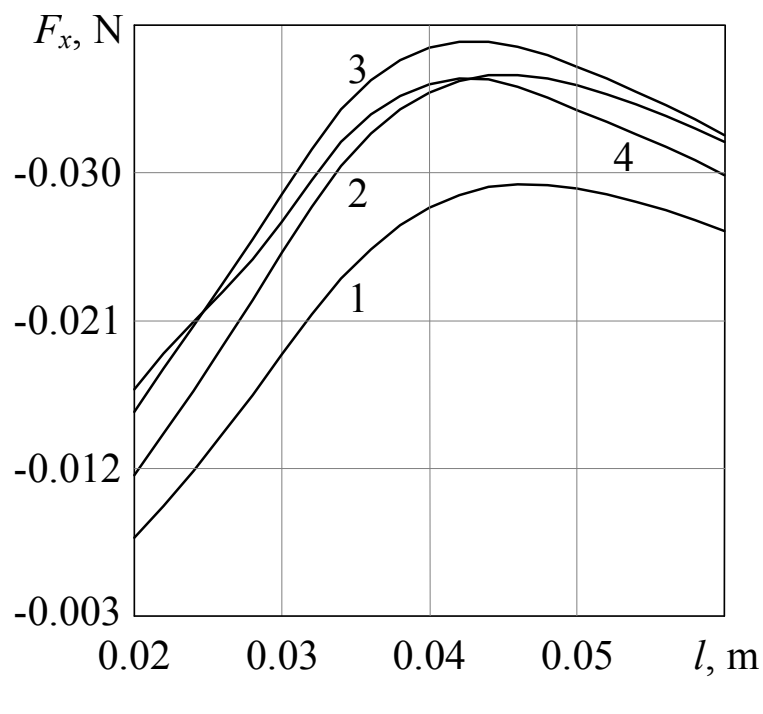

$a$

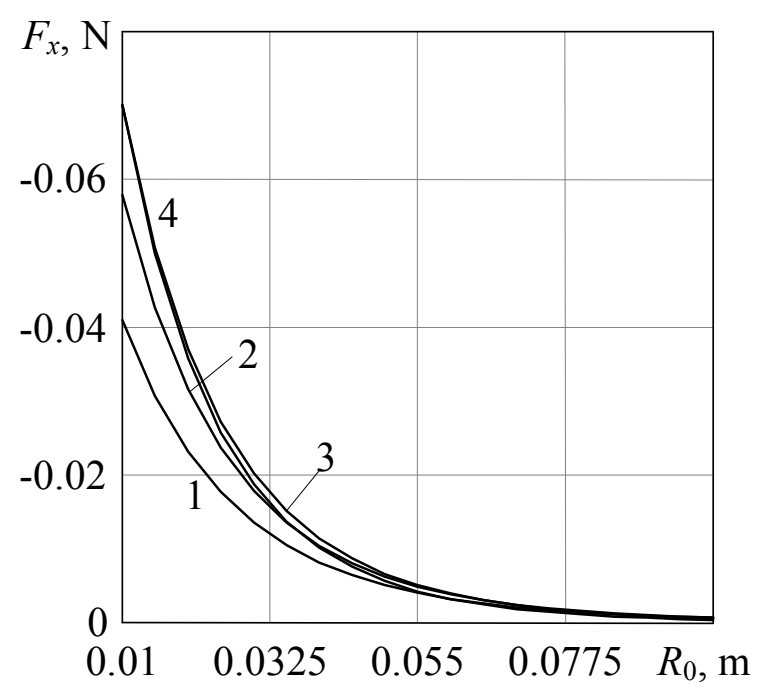

$\sigma$

Pис. 5. Зависимость горизонтальной компоненты сил от расстояния между стержнями $d$ $\left(R_{0}=25 \mathrm{мм}\right)$ (а) и расстояния до металла $R_{0}(d=25$ мм) (б) при разной частоте тока: $1-25 \Gamma u, 2-50$ Гu, $3-100 \Gamma u, 4-250$ Гu

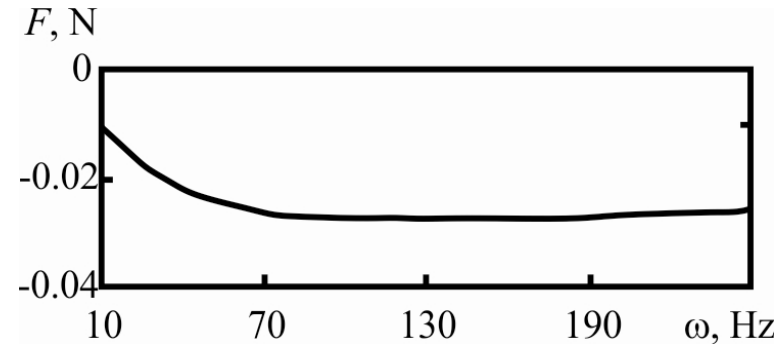

Рис. 6. Зависимость горизонтальной компоненты электромагнитной силь, действующей на металл в ванне модели, от частоты переменного тока ш. Расстояние между шинами $d=25$ мм, расстояние от иин до металла $R_{0}=25$ мм

устойчивый вихрь с небольшими неустойчивыми вихрями около стенок и в углах ванны. Течение имеет максимальную интенсивность в придонной области (рис. 7).

4. Перемешивание в ванне внесенной примеси. Для моделирования перемешивания пассивной примеси (рис. 8) в

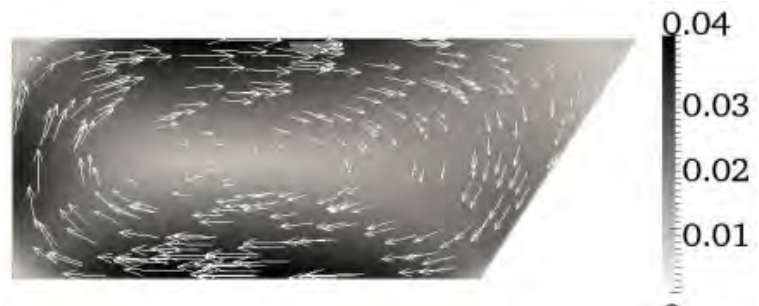

Рис. 7. Векторное поле скорости (икала значений скорости в $м /$ ) при токе в индукторе 200 А и частоте тока 15 Ги ванне модели при включении электромагнитных сил использовались уравнения движения и уравнение Фике:

$$
\partial C / \partial t+(V \nabla) C=\nabla \cdot(D \nabla C) .
$$

Как показывают расчеты, в начальный момент времени идет интенсивное размывание примеси. Появившиеся вихри перемешивают примесь. Происходит формирование крупномасштабного вихря. Далее, при установившемся течении примесь начинает переноситься как одно целое и ее размывание замедляется (так как происходит в основном за счет диффузионного механизма). Для определения степени гомогенизации сплава мы ввели и предлагаем использовать параметр $\xi(t)$ («коэффициент неоднородности»), который имеет смысл среднеквадратичного отклонения концентрации примеси $C$ во всех $n$ точках расчетной области от конечной концентрации, после идеального перемешивания (в момент времени $t_{1}$ ) к среднеквадратичному отклонению в начальный момент времени $t=0$. Введем также параметр $t_{\text {eff }}-$ «характерное время перемешивания», который связывает затраченное на перемешивание время с достигнутой степенью гомогенизации и равен интервалу времени, за который $\xi$ уменьшается в 10 раз: 


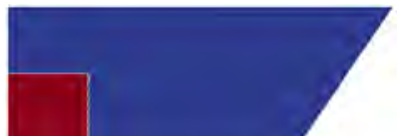

$a$

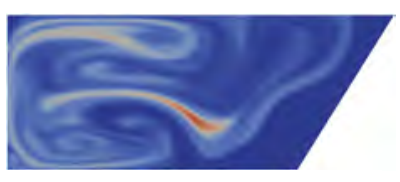

2

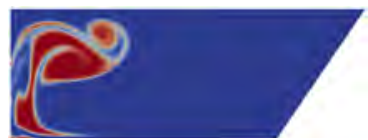

$\sigma$

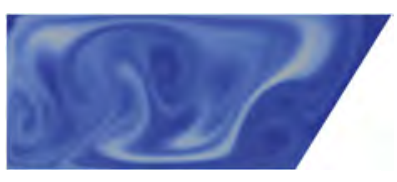

$\partial$

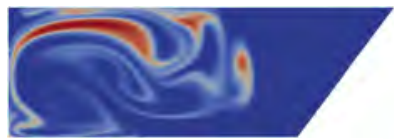

B

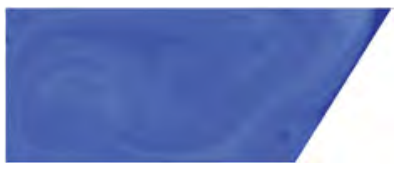

$e$

Рис. 8. Эволюция распределения примеси (изначально квадрат в правом нижнем углу) в ванне $I=200$ A, время: а) 0; б) $10 \mathrm{c}$; в) $60 \mathrm{c}$; г) $120 \mathrm{c}$; д) $200 \mathrm{c}$; е) $400 \mathrm{c}$

$\xi\left(t_{1}\right)=$

$=\frac{\sqrt{\left.\frac{1}{n}\left\{\left(\bar{C}-C_{1}\right)^{2}+\left(\bar{C}-C_{2}\right)^{2}+\ldots+\left(\bar{C}-C_{n}\right)^{2}\right\}\right|_{t=11}}}{\sqrt{\left.\frac{1}{n}\left\{\left(\bar{C}-C_{1}\right)^{2}+\left(\bar{C}-C_{2}\right)^{2}+\ldots+\left(\bar{C}-C_{n}\right)^{2}\right\}\right|_{t=0}}}$.

Как видно на рис. 9, зависимость характерного времени перемешивания от частоты имеет минимум в области $100-$ 140 Гц (минимальное время перемешивания) и величину, близкую к постоянному значению на низких частотах.

Интервал частот для минимума времени перемешивания совпадает с частотами, при которых электромагнитная сила, действующая на металл в ванне, максимальна (см. рис. 6).

5. Реверсный режим. Реверсный режим при воздействии на жидкий металл вращающегося магнитного поля рассматривался в $[5,6,9]$. В нашей работе мы применяли его в приложении к бегущему магнитному полю. Для электромагнитных сил использовали коробчатую зависимость от времени:

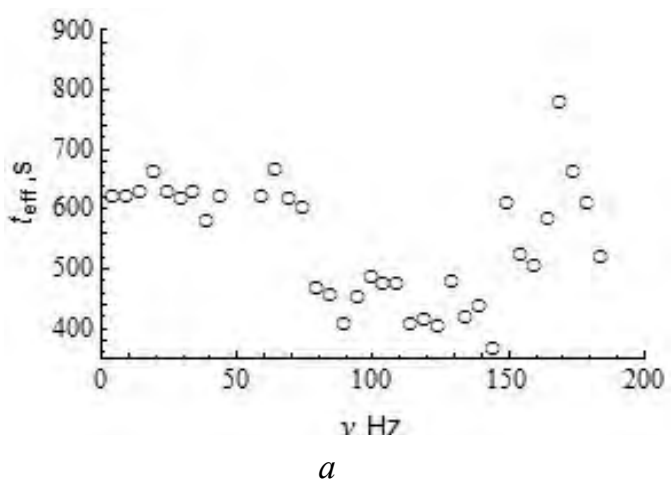

$$
F=F_{a} \cdot \theta(\varsigma), \varsigma=\sin \left(\omega_{0} t\right), \theta(\varsigma)=\left\{\begin{array}{c}
1, \varsigma>0 \\
-1, \varsigma \leq 0
\end{array}\right.
$$

Как показали численные эксперименты, реверсный режим на различных частотах реверса может как увеличивать, так и сильно снижать время перемешивания. Как можно видеть на рис. 10 и таблицы, при частоте бегущего поля 100 Гц его реверс с частотой 0,08 Гц существенно снижает время перемешивания в сравнении с другими режимами реверса и с режимом его отсутствия.

Анализ результатов численных экспериментов показал, что для улучшения процесса перемешивания необходимо создавать режимы с неустойчивыми вихревыми структурами, важными также являются интенсивность течения и масштабы возникающих вихрей. Можно выделить две функции вихрей в процессе перемешивания. Первая функция - конвективная, при которой главную роль играют вихри крупного масштаба, при этом происходит захват частей примеси и перенос

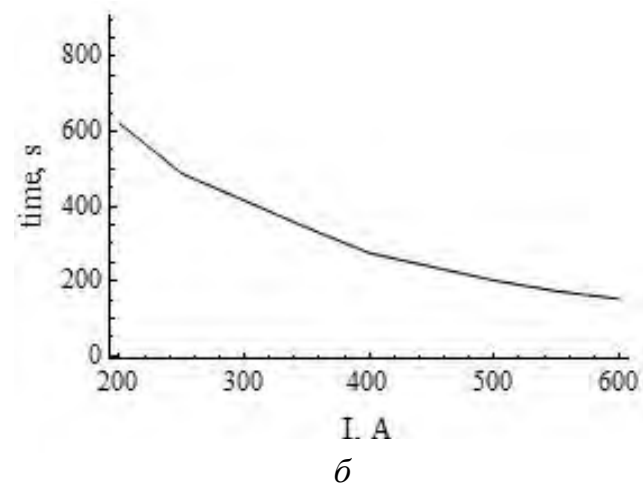

Рис. 9. Зависимость характерного времени перемешивания: а) от частоты тока в индукторе $\left(I_{0}=200 \mathrm{~A}\right)$; б) от силь тока в индукторе $(v=15$ Ги) 


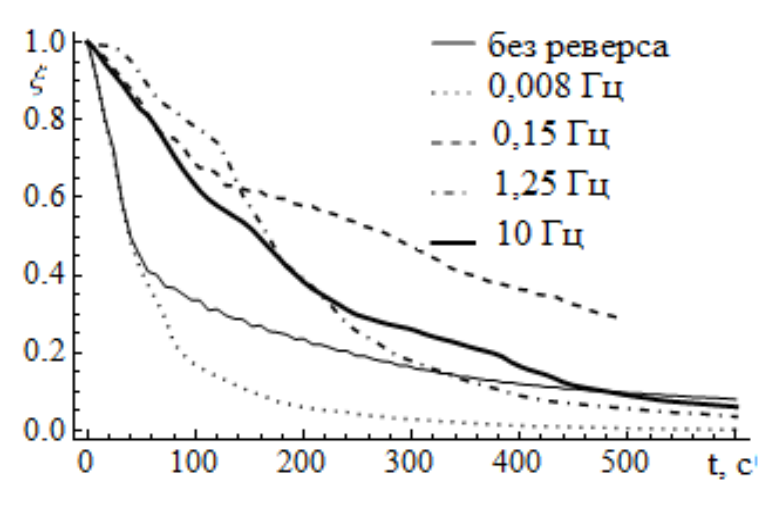

Рис. 10. Зависимость параметра перемешивания при реверсивном режиме от времени перемешивания для различных частот реверса и частоты тока в индукторе (100 Ги)

\begin{tabular}{|c|c|c|c|}
\hline $\begin{array}{c}\text { Частота } \\
\text { реверса, Гц }\end{array}$ & $\begin{array}{c}\text { |Vmax|, } \\
\text { см/c }\end{array}$ & $\begin{array}{c}\text { |Vсредн|, } \\
\text { см/c }\end{array}$ & $\begin{array}{c}\text { Время } \\
\text { перемеши- } \\
\text { вания, c }\end{array}$ \\
\hline без реверса & 5,4 & 2,1 & 432 \\
\hline 0,008 & 4,0 & 1,2 & 150 \\
\hline 0,15 & 1,3 & 0,4 & 781 \\
\hline 1,25 & 0,6 & 0,3 & 387 \\
\hline 10,000 & 1,2 & 0,2 & 481 \\
\hline
\end{tabular}

их в места обедненные примесью. Вторая функция - диффузионная, при которой происходит «растворение» примеси, здесь основную роль играет механизм турбулентной диффузии, который обусловлен главным образом вихрями мелкого масштаба.

Реверсный режим с одной стороны приводит к возникновению нестабильных вихревых образований, что улучшает процесс переноса примеси, но с другой стороны с увеличением частоты реверса снижается интенсивность течения, что ухудшает процессы переноса. Таким образом, существуют только определенные частоты реверса, при которых процесс перемешивания идет с наилучшей скоростью.

\section{6. Результаты экспериментов и оцен-} ки параметров перемешивания для реальных печей. В экспериментах на «галлиевой» модели допплеровским анемометром измерялись скорости жидкого металла во многих точках внутри ванны модели при разных режимах перемешивания (рис. 11). На «галлиевой» и «алюминиевой» моделях термопарным зондом опре- делялось поведение вертикального градиента температуры в жидком металле при различных режимах перемешивания. Как показал эксперимент на жидком галлии, течение в кювете похоже на расчетное течение, но носит более сложный характер. В продольном сечении существует один крупный (масштаба ванны) вихрь, в то же время в поперечном сечении возбуждается двухвихревое течение, обусловленное главным образом наличием трения жидкости о боковые вертикальные стенки ванны и неоднородностью магнитного поля вдоль токоведущих стержней индуктора, связанной с их ограниченностью по длине.

Течение, возбуждаемое в ванне индуктором, характеризуется безразмерными числами $S=f \cdot h^{3} / \rho v^{2}=j_{0} B_{0} h^{3} / \rho v^{2}=$ $=\sigma \omega \mu_{0}^{2} h^{4} I_{0}^{2} / 4 \rho v^{2} \pi^{2} R_{0}^{2}$ и $\delta=h^{2} \sigma \mu_{0} \omega$. Приравняем числа $S$ и $\delta$ для лабораторной алюминиевой ванны и для галлиевой модели (при токе индуктора 238 А (93 Гц) в галлиевой модели скорость $3,3 \mathrm{~cm} / \mathrm{c})$.

$$
\begin{aligned}
& I_{A l}^{2} \sigma_{A l} \omega \mu_{0}^{2} h_{A l}^{4} / 4 \rho_{A l} \nu_{A l}^{2} \pi^{2} R_{0 A l}^{2}= \\
& =I_{G a}^{2} \sigma_{G a} \omega \mu_{0}^{2} h_{G a}^{4} / 4 \rho_{G a} \nu_{G a}^{2} \pi^{2} R_{0 G a}^{2}, \\
& h_{A l}^{2} \sigma_{A l} \mu_{o} \omega_{A l}=h_{G a}^{2} \sigma_{G a} \mu_{o} \omega_{G a},
\end{aligned}
$$

где $\sigma_{A l}=3,4 \cdot 10^{6} \mathrm{CM} / \mathrm{M}, \sigma_{G a}=3,56 \cdot 10^{6} \mathrm{CM} / \mathrm{M}$, $h_{A l}=0,140 \mathrm{M}, h_{G a}=0,1 \mathrm{M}, \rho_{A l}=2,32 \cdot 10^{3} \mathrm{\kappa} / \mathrm{m}^{3}$, $\rho_{G a}=6,256 \cdot 10^{3} \mathrm{\kappa} \Gamma / \mathrm{m}^{3}, \quad v_{A l}=0,86 \cdot 10^{-6} \quad \mathrm{~m}^{2} / \mathrm{c}$, $v_{G a}=0,31 \cdot 10^{-6} \mathrm{M}^{2} / \mathrm{c}, \omega_{G a}=314, R_{0 A}=0,165 \mathrm{M}$, $R_{0 G a}=0,025 \mathrm{M}$.

Получим, что такие же гидродинамические условия будут в алюминиевой ванне при токе индуктора 1900 А и частоте 50 Гц. В этом случае будем считать, что в алюминиевой ванне безразмерная величина скорости жидкого металла (число Рейнольдса) будет близко к скорости металла в галлиевой модели:

$$
V_{G a} \cdot h_{G a} / v_{G a}=V_{A l} \cdot h_{A l} / v_{A l} .
$$

Из этого условия найдем оценку скорости металла в алюминиевой ванне в эксперименте. При токе 1900 А и частоте 50 Гц она будет $6,5 \mathrm{~cm} / \mathrm{c}$.

На основе полученных оценок определим величину максимальной скорости МГД-перемешивания в ванне промыш- 

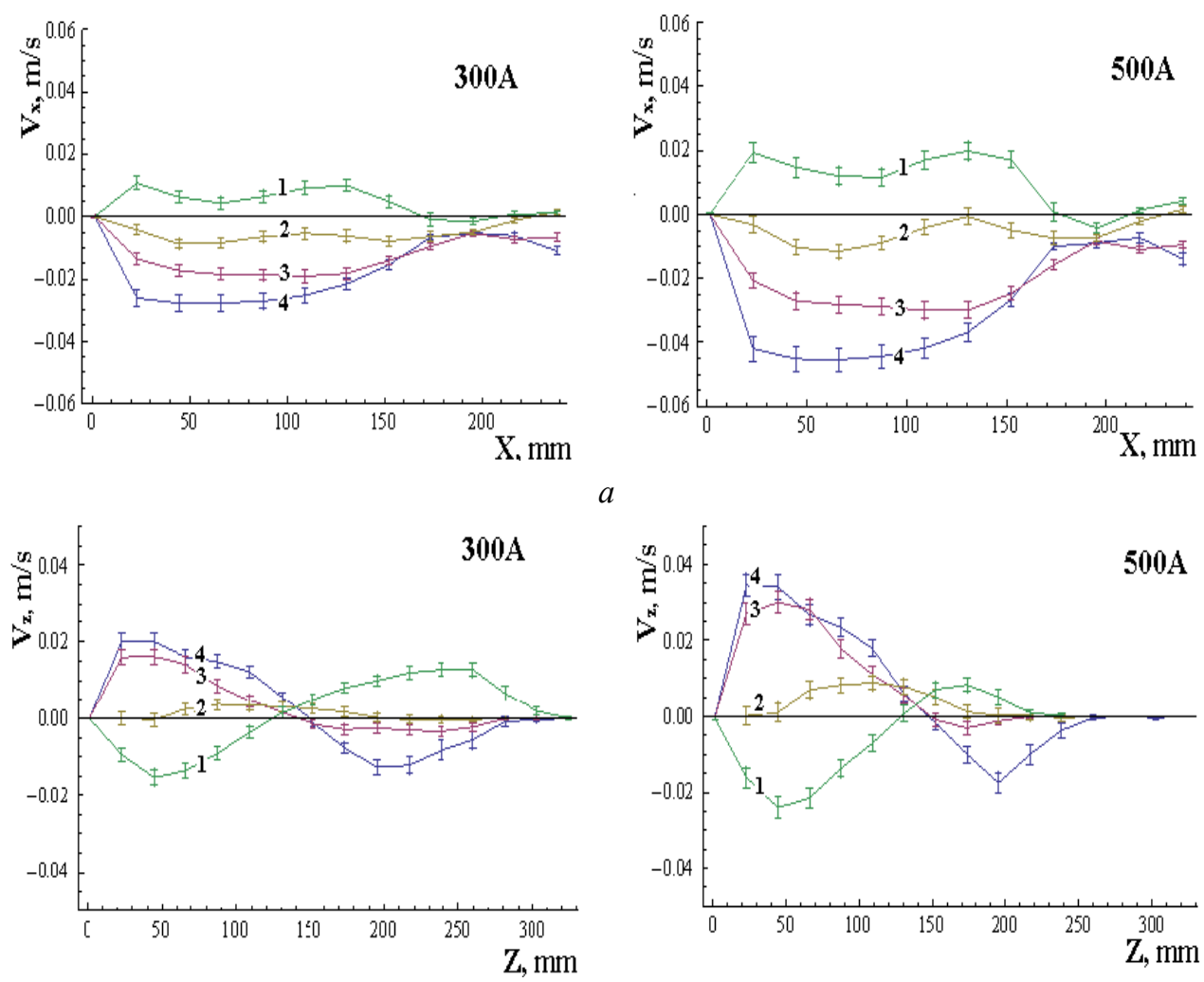

$\sigma$

Рис. 11. Распределение скоростей жидкого металла (галлия) в ванне физической модели вдоль (а) и поперек (б) скорости бегущего поля для тока в индукторе $300 \mathrm{~A}$ и 500 А (50 Гч). Цифрами в порядке возрастания помечены экспериментальные кривые, полученные от датчиков скорости, расположенных вертикально на боковых стенках (см. рис. 1). Верхнему датчику соответствует кривая 1

ленной алюминиевой печи (см. рис. 1).

Приравняем числа $S_{A l}$ и $\delta_{A l}$ для алюминиевой ванны в лабораторном эксперименте и числа $S_{F}$ и $\delta_{F}$ для ванны промышленной алюминиевой печи:

$$
\begin{aligned}
& I_{A l}^{2} \sigma_{A l} \omega_{A l} \mu_{0}^{2} h_{A l}^{4} / 4 \rho_{A l} v_{A l}^{2} \pi^{2} R_{0 A l}^{2}= \\
& =I_{F}^{2} \sigma_{F} \omega_{F} \mu_{0}^{2} h_{F}^{4} / 4 \rho_{F} v_{F}^{2} \pi^{2} R_{0 F}^{2}, \\
& h_{A l}^{2} \sigma_{A l} \mu_{0} \omega_{A l}=h_{F}^{2} \sigma_{F} \mu_{0} \omega_{F} .
\end{aligned}
$$

Получим, что подобные условия будут в промышленной ванне при токе индуктора 541 А и частоте 12,5 Гц. Скорость металла при этом будет $1,8 \mathrm{~cm} / \mathrm{c}$. Учитывая то, что зависимость скорости перемешивания от силы тока в индукторе близка к линейной (рис. 12), получим, что при токе в индукторе 3000 А и частоте 12,5 Гц скорость перемешивания в промышленной ванне составит $10 \mathrm{~cm} / \mathrm{c}$.

Такая частота не совсем удобна для источников питания, но, как показывает физический эксперимент (рис. 13), продольная скорость перемешивания несильно зависит от частоты; это позволяет надеяться, что в индукторах промышлен-

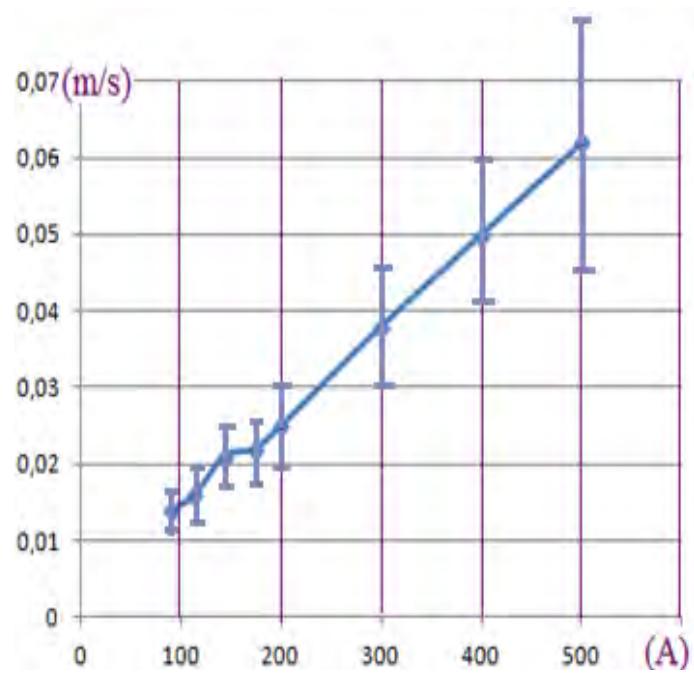

Рис. 12. Зависимость максимальной скорости жидкого галлия в ванне (физической модели) от тока в индукторе (50 Ги) 


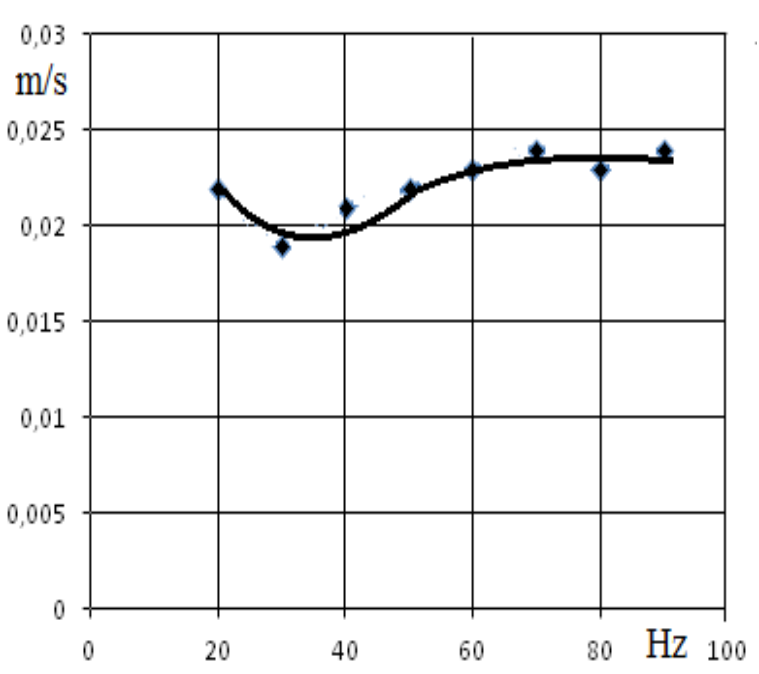

Рис. 13. Зависимость максимальной продольной скорости галлиевого сплава (15 мм от дна ванны) от частоты тока в индукторе (ток 174 A)

ных печей можно использовать ток промышленной частоты без значительного снижения эффективности.

Будем считать временем перемешивания то, за которое вертикальный градиент температуры снижается в $e$ раз. Как показали численный (рис. 14) и физический (рис. 15) эксперименты, время перемешивания резко падает, начиная с некоторого

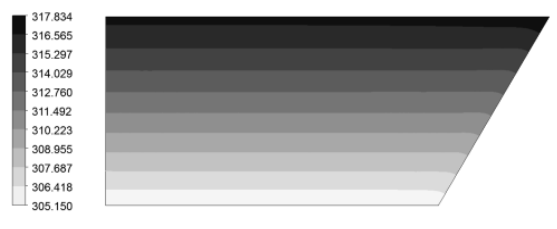

$a$
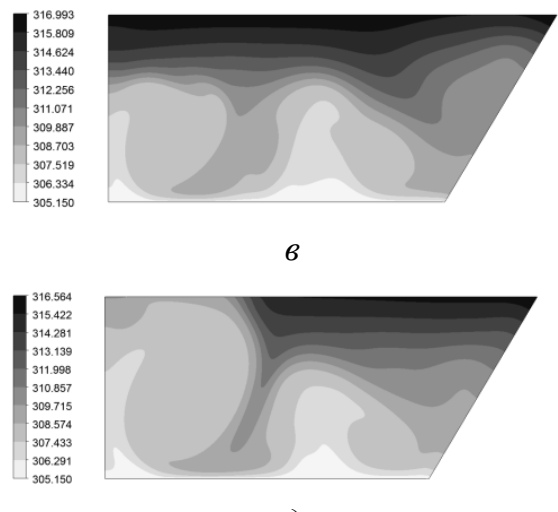

$\partial$ порогового значения тока в индукторе (рис. 16). До этого порога (по току) градиент температуры меняется слабо и время перемешивания неопределенно.

Заключение. Как показали физические и численные эксперименты, а также оценки, полученные из критериев физического подобия, при помощи индуктора предложенной конструкции можно создавать перемешивающие течения, способные выровнять температуру жидкого сплава не только в ваннах физических моделей, но и в ваннах промышленных алюминиевых печей. Было обнаружено, что резкое уменьшение температурного градиента в ванне наступает после некоторого порогового значения тока в индукторе (см. рис. 16). При увеличении частоты фиксированного по величине тока (170 А) время перемешивания в галлиевой модели резко падает и становится минимальным при частоте около 50 Гц (рис. 17). При дальнейшем увеличении частоты время перемешивания медленно увеличивается. Этот экспериментальный факт позволяет надеяться, что для больших промышленных печей, для которых наилучшими являются пониженные час-
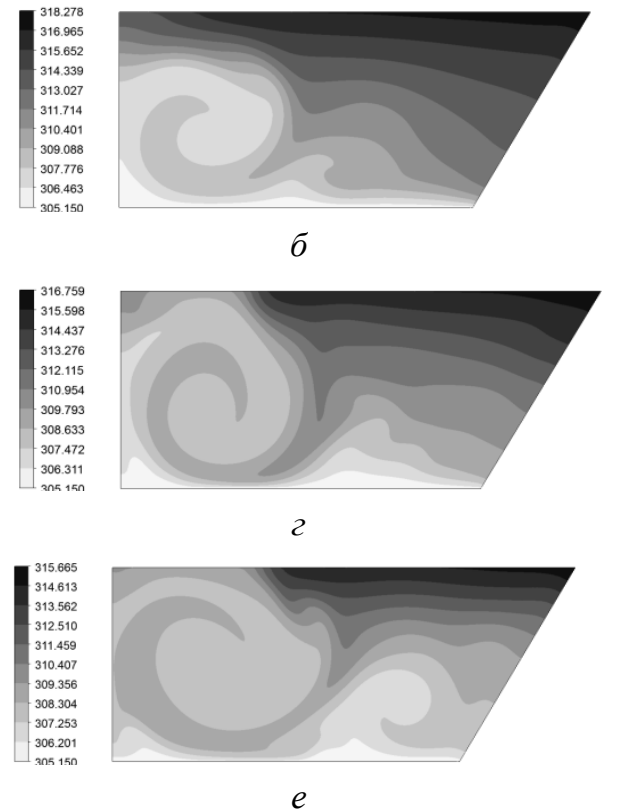

Рис. 14. Эволючия поля температур с течением времени под действием МГД-перемешивания при токе в индукторе 170 А, 50 Ги:: а-начальный момент, $6-20 c$, в-40 c, г-60 c, $\partial-80 c, e-100$ c. Время перемешивания в эксперименте составило $73,8 \mathrm{c}$ 


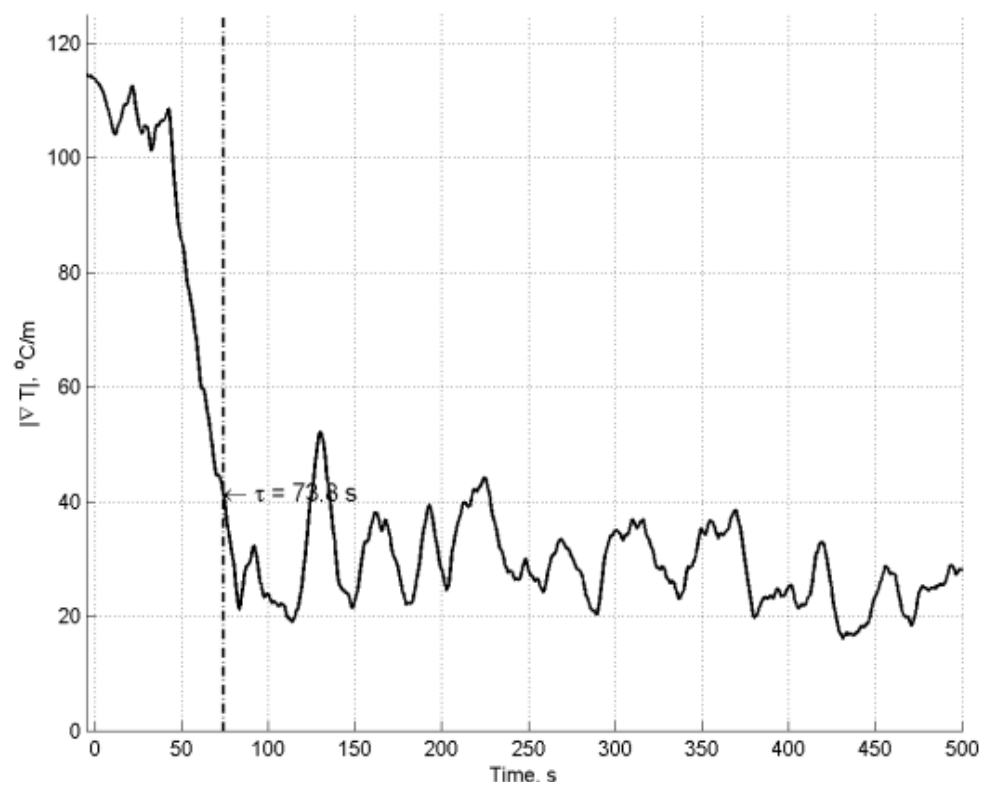

Рис. 15. Эволюция вертикального градинта температур в галлиевой модели с течением времени под действием МГД-перемешивания при токе в индукторе 170 А, 50 Ги. Время перемешивания в эксперименте составило 73,8 с

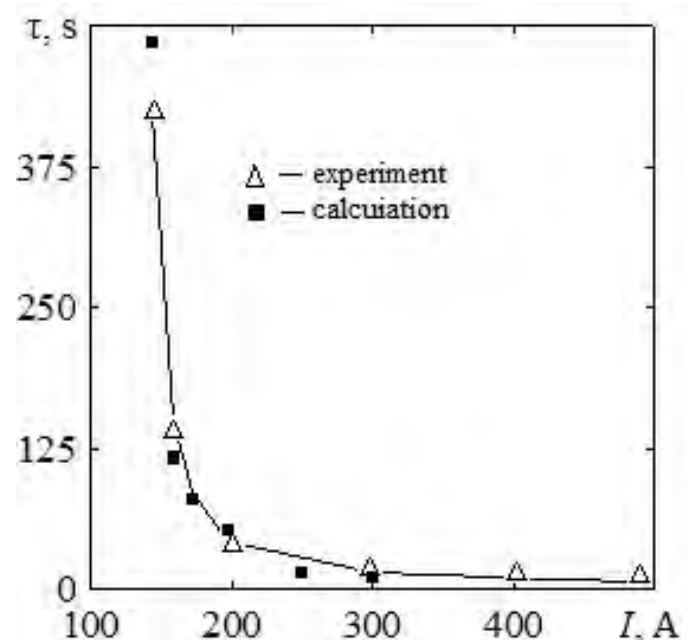

Рис. 16. Изменение времени перемешивания сплава с ростом тока в индукторе. Частота тока 50 Ги (результаты расчетов и эксперимента на галлиевой модели)

тоты, можно будет так же использовать токи промышленной частоты (50 Гц) без больших потерь в эффективности. В численных экспериментах было показано, что МГД-перемешивание наиболее эф-

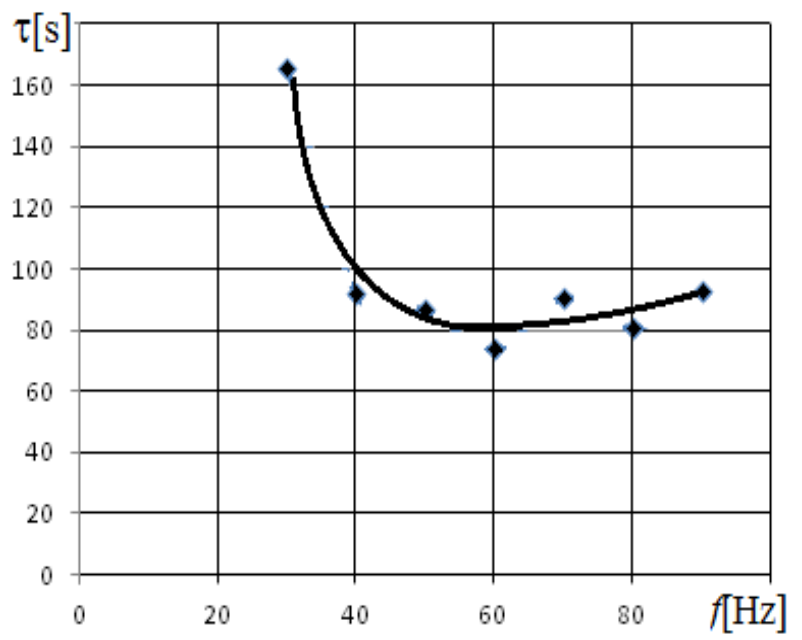

Рис. 17. Изменение времени перемешивания сплава с ростом частоты тока в индукторе. Сила тока в индукторе 170 A (эксперимент на галлиевой модели)

фективно в реверсном режиме, причем существуют определенные частоты реверса поля, при которых перемешивающий эффект максимален.

\section{Библиографический список}

1. Волков К.Н. Реализация схемы расщепления на разнесенной сетке для расчета нестационарных течений вязкой несжимаемой жидкости // Вычислительные методы и программирование. - 2005. T. 6. - № 1. - C. 269-282. 
2. Оборин П.А., Хрипченко С.Ю. Генерация течения жидкого металла и перенос пассивной примеси в прямоугольной полости бегущим магнитным полем // Вычислительная механика сплошных сред. 2013. - Т. 6. - № 2. - С. 207-213.

3. Франк-Каменеикий Д.А. Диффузия и теплопередача в химической кинетике. - М.: Наука, 1967. -492 с.

4. Хрипченко С.Ю., Оборин П.А. Патент (Российской Федерации) RU 2567970 C1 от 05.08.2014. Дата публикации: 10.11.2015. Бюлл. № 31.

5. Eckert $S$. [et al.] Efficient melt stirring using pulse sequences of a rotating magnetic field: Part i. flow field in a liquid metal column // Metallurgical and Materials Transactions B. - 2007. - Vol. 38. - № 6. - P. 977-988.

6. Lei Z.S., Yang X.H., Wei R.J., Gao Q. A new criterion for optimization the intermittently reversing direction electromagnetic stirring in round strands continuous casting // Advanced Materials Research. 2013. - Vol. 785. - P. 1095-1098.

7. Oborin P., Khripchenko S. Simulation study on the applicability of travelling magnetic fields in metallurgical furnaces for reducing temperature difference in the molten metal // Russian conference on Magnetohydrodynamics RMHD-2015. - Perm, Russia, 2015. Book of Abstracts. - P. 70.

8. Ščepanskis M., Jakovičs A., Nacke B. Homogenization of non-conductive particles in EM induced metal flow in a cylindrical vessel // Magnetohydrodynamics. - 2010. - Vol. 46. - № 4. - P. 413-423.

9. Wang X., Moreau R., Etay J., Fautrelle Y. A periodically reversed flow driven by a modulated traveling magnetic field: Part ii. theoretical model // Metallurgical and Materials Transactions B. - 2009. - Vol. 40. № 1. - P. 104-113.

\title{
DEVELOPMENT AND CREATION OF A LABORATORY MODEL OF AN INDUSTRIAL OVEN WITH MHD-STIRING FOR ALUMINUM AND ITS ALLOYS
}

\author{
S.Yu. Khripchenko, S.A. Denisov, V.M. Dolgikh, S.V. Lekomtsev, A.M. Pavlinov, P.A. Oborin \\ Institute of Continuous Media Mechanics UB RAS
}

In this work using physical models of an aluminum furnace bath, the mixing process of a liquid metal with a traveling magnetic field, which is created by a system of parallel linear conductors located under the bottom of the bath, is investigated. The flow velocity profiles and the dependence of the mixing rate on the force and frequency of the current in the inductor were obtained on a gallium model using a Doppler anemometer. In the experiment, a sharp decrease in the vertical temperature gradient in the bath was found when the threshold value of the mixing rate of the liquid metal was reached. With the help of mathematical modeling, the flow pattern, the evolution of the temperature field and the spread of the impurities in the bath of the physical model under MHD stirring are calculated. The results are compared with the results of the physical experiments.

Keywords: aluminum furnace, MHD stirring, traveling magnetic field of a linear inductor, passive impurity, mixing time, temperature gradient.

\section{Сведения об авторах}

Хрипченко Станислав Юрьевич, доктор технических наук, ведущий научный сотрудник лаборатории физической гидродинамики, Институт механики сплошных сред УрО РАН (ИМСС УрО РАН), 614013, г. Пермь, ул. Академика Королева, 1; e-mail: khripch@icmm.ru

Денисов Сергей Анатольевич, кандидат технических наук, старший научный сотрудник лаборатории физической гидродинамики, ИМСС УрО РАН; e-mail: denisov@icmm.ru

Долгих Вениамин Михайлович, кандидат технических наук, старший научный сотрудник лаборатории физической гидродинамики, ИМСС УрО РАН; e-mail: Dolgikh @icmm.ru

Лекомиев Сергей Владимирович, кандидат физико-математических наук, научный сотрудник отдела комплексных проблем механики деформируемых твердых тел, ИМСС УрО РАН; e-mail: lekomtsev@icmm.ru

Павлинов Александр Михайлович, аспирант лаборатории физической гидродинамики, ИМСС УрО PAH; e-mail: pam@icmm.ru

Оборин Павел Александрович; e-mail: khripch@icmm.ru 